\title{
Faraday rotation in the CSS QSOs 3C 43 and 3C 454
}

\author{
W. D. Cotton ${ }^{1}$, R. E. Spencer ${ }^{2}$, D. J. Saikia ${ }^{3}$, and S. Garrington ${ }^{2}$ \\ 1 National Radio Astronomy Observatory, 520 Edgemont Road, Charlottesville, VA 22903-2475, USA \\ 2 NRAL-Jodrell Bank, University of Manchester, Macclesfield Cheshire, SK11 9DL, UK \\ 3 Tata Institute of Fundamental Research, National Centre for Radio Astrophysics, Pune 411 007, India
}

Received 18 November 2002 / Accepted 28 February 2003

\begin{abstract}
We present observations of the CSS QSOs 3C 43 and 3C 454 made with the VLA, MERLIN and VLBA at 2, 6 and $18 \mathrm{~cm}$ to examine the Faraday rotation in these highly bent sources. 3C 43 is weakly polarized at $18 \mathrm{~cm}$ at mas resolution but has a large rotation measure near the bend in the jet. This result is interpreted as indicating jet-ISM interaction. 3C 454 is relatively strongly polarized and has high rotation measure along the jet visible in the high resolution $18 \mathrm{~cm}$ images. There is no brightening of the jet near the bend to the west indicating that it is not caused by a jet-cloud collision and may simply be a hydrodynamic instability magnified by a small angle to the line of sight. However, there are clear differences in the sign and magnitude of the Faraday rotation measured at long and short wavelengths. This is interpreted as being due to thermal plasma mixed with the jet. Likely detection of circular polarization at $18 \mathrm{~cm}$ supports this interpretation.
\end{abstract}

Key words. polarization - galaxies: quasars: individual: 3C 43, 3C 454 - galaxies: jets - galaxies: nuclei radio continuum: galaxies

\section{Introduction}

Depending on the frequency of observation, approximately $15-30 \%$ of all strong radio sources selected at low frequencies (Fanti et al. 1995) are classified as Compact Steep Spectrum (CSS) sources (Kapahi 1981; Peacock \& Wall 1982). These are powerful radio sources with apparent sub-galactic linear dimensions and steep spectra $\left(\alpha \leq-0.5, S \propto v^{\alpha}\right)$ at high frequencies. Due to the small sizes and high powers of these sources, they are suspected of being the progenitors of the extended double radio sources. Their angular dimensions are of the order of a few arcseconds and they are related as a class to the more compact Giga-Hertz Peaked Sources (GPS). A detailed review is given by O'Dea (1998).

Fanti et al. (1990) argue that only a small fraction $(<25 \%)$ of CSS sources are larger sources oriented close to the line of sight. Most of the objects are intrinsically small, and are either "young" ( $~ 10^{6}$ years), or "frustrated", where the size is limited by strong interactions of their jets with interstellar gas. Fanti et al. (1995) and Readhead et al. (1996) find that the youth scenario is strongly supported by evidence from the radio structures. However, the presence of dense interstellar gas is known from optical observations. Gelderman \& Whittle (1994) found high forbidden line luminosities and interpreted their results as indicating jet-ISM interactions. The HST narrow band imaging results of Axon et al. (2000) show several CSS sources where the emission line gas extends along the radio axis and

Send offprint requests to: W. D. Cotton, e-mail: bcotton@nrao. edu beyond the size of the radio source, further supporting such interactions.

Magnetized plasma capable of confining or deflecting the radio jets can also be detected by means of Faraday rotation of the linearly polarized emission from the jet. Indeed, a recent multi-frequency high resolution study of the jet in the CSS source 3C 138 (Cotton et al. 1997) shows that the rotation measure of the jet is only 10 's of $\mathrm{rad} \mathrm{m}^{-2}$. However, the core is strongly depolarized at $18 \mathrm{~cm}$ and VLBA measurements of the core using bands separated by $300 \mathrm{MHz}$ at $5 \mathrm{GHz}$ show that the rotation measure is around $-1800 \mathrm{rad} \mathrm{m}^{-2}$. Thus, the core is behind dense ionized gas containing a magnetic field, whereas the relatively straight jet is not. The youth scenario seems to fit here. High rotation measures have also been found in the cores of more extended quasars (Taylor 1998).

On the other hand, there are CSS sources whose jets are clearly bent, in some cases by greater than 90 degrees, apparently by interaction with interstellar gas, even if projection effects magnify the apparent degree of bending. These bent sources are mostly quasars (Spencer et al. 1991; Mantovani et al. 1998); for example 3C 147 has an asymmetric jet with high rotation measure on one side, probably caused by interaction with ionized gas (Junor et al. 1999).

If these jets have been deflected by initially neutral ISM gas, there should be concentrations of plasma at the impact point. Since these bends are in the main (presumably approaching) jets of QSOs, our line of sight should pass through this jet-ionized gas, increasing the Faraday rotation of emission from the region of the bend. 
Previous attempts to look for enhanced Faraday rotation around the bends in CSS jets have been made by Mantovani et al. (2002) who studied the bent jet CSS sources B0548+165 and B1524-136 using the VLBA in 5 wavelength bands at 6 and $3.6 \mathrm{~cm}$. They found large rotation measures (1000-10000 rad m-2) in both jets with enhanced Faraday rotation near the bend in $\mathrm{B} 0548+165$ but not near that in B1524-136.

In the present study, we examine two sources $(3 \mathrm{C} 43=$ $\mathrm{J} 0129+236$ and $3 \mathrm{C} 454=\mathrm{J} 2251+188)$ from a sample of CSS sources observed with MERLIN by Lüdke et al. (1998). These sources have particularly sharp bends in their jets and polarization at the level of a few percent at $5 \mathrm{GHz}$. Based on VLA and MERLIN observations at $6 \mathrm{~cm}$, Akujor et al. (1991b) suggested that the jet in 3C 43 had been deflected by the ISM. The sources have also been previously studied by Spencer et al. (1991) with EVN + MERLIN observations at $18 \mathrm{~cm}$, and by Akujor et al. (1991a) with MERLIN observations at $6 \mathrm{~cm}$. With the Nobeyma telescope, Inoue et al. (1995) detected integrated polarization at $3 \mathrm{~cm}$ wavelength of $2.5 \%$ in $3 \mathrm{C} 43$ and $9.9 \%$ in $3 \mathrm{C} 454$ but could only set $2 \sigma$ upper limits to their rotation measures of 1280 and $664 \mathrm{rad} \mathrm{m}^{-2}$ respectively. Fanti et al. (2002) discuss a multi-frequency analysis of 3C 43 using MERLIN and EVN observations. The morphology of 3C 43 is decribed in detail in that paper.

$3 \mathrm{C} 43$ is a QSO at $z=1.46 ; 3 \mathrm{C} 454$ is also a quasar at a redshift of 1.7578. Lehnert et al. (1999) detect extended optical emission around the nuclei of both these objects using broadband filters on the HST, although these observations do not distinguish between starlight and emission from plasma in the ISM. de Vries et al. (1999) made snapshot images of 3C 43 and $3 \mathrm{C} 454$ in narrow band filters using the HST but were unable to determine if emission line gas was coextensive with the radio source due to the strong nuclear component.

Based on spectral aging estimates, Murgia et al. (1999) estimate extremely young ages for both of these sources, 300 years for 3C 43 and 800 years for 3C 454. These ages give rather implausibly high expansion rates. However, Fanti et al. (2002) estimate a typical age for CSS sources of $\sim 3 \times 10^{5}$ years from more complete spectral data from high resolution images. In the present paper we use the following: $H_{0}=65 \mathrm{~km} \mathrm{~s}^{-1} \mathrm{Mpc}^{-1}$, $\Omega_{\text {Matter }}=0.3$ and $\Omega_{\Lambda}=0.7$ for which one arcsecond at either source is $9.1 \mathrm{kpc}$.

\section{Observations}

3C 43 and 3C 454 were observed at $2 \mathrm{~cm}$ with the VLA in "A" configuration, at $6 \mathrm{~cm}$ with the MERLIN array, and at $18 \mathrm{~cm}$ with the VLBA plus a VLA antenna to examine the polarization and rotation measures in these objects.

\subsection{VLA $2 \mathrm{~cm}$}

The VLA observations were made on 2 December 2000 and used two $50 \mathrm{MHz}$ bands centered at $14.96(2.00 \mathrm{~cm})$ and $14.91(2.01 \mathrm{~cm}) \mathrm{GHz}$. Right- and left-hand circular polarizations were used and all four correlation products were retained. All processing was done in the NRAO AIPS package following standard procedures. Flux density calibration was based on 3C 48 (1.81 Jy). Astrometric and instrumental polarization calibration was based on $\mathrm{J} 0122+250$ for $3 \mathrm{C} 43$ and $\mathrm{J} 2253+161$ for $3 \mathrm{C} 454$. Polarization angle calibration used $3 \mathrm{C} 138$ with an assumed polarization angle of $-12.0^{\circ}$.

\subsection{MERLIN $6 \mathrm{~cm}$}

The MERLIN observations were taken on 7 Dec. 1992 (3C 43) and 16 April 2001 (3C 454) at $4.993 \mathrm{GHz}(6.0 \mathrm{~cm})$. The flux density scale for both sources was established from observations of 3C 286 made closely in time to the target observations using the three short baselines between the Mk2, Tabley and Darnhall telescopes. The assumed flux density of 3C 286 was $7.006 \mathrm{Jy}$ which includes corrections for resolution on the shortest MERLIN baselines. These data were used to find the flux density of the point source OQ208 (2.49 Jy) used to calibrate the baselines for 3C 43, and J0555+398 (6.10 Jy) used to calibrate the data for $3 \mathrm{C} 454$, after correcting for variations of the telescope gains with elevation. Phase reference observations were made for each target source, the reference sources being $\mathrm{J} 0145+233$ for $3 \mathrm{C} 43$ and $\mathrm{J} 2250+211$ for $3 \mathrm{C} 454$. The above analysis was performed using the " $\mathrm{d}$ " programs specially written for MERLIN data. Some editing and flagging of poor data was performed at this stage.

Subsequent analysis was done in the AIPS package. This performed the delay-rate calibration, right-left delay calibration corrections, and made a bandpass correction after dropping the first and last of the 15 channels from the correlator. Instrumental polarization calibration was done using the (unpolarized) point source calibrator. The polarization angle calibration was initially performed using 3C 286 and an assumed polarization angle of $33^{\circ}$. The summed CLEAN Stokes $Q$ and $U$ components from a self-calibrated image were used for this calibration. The relative phase of the two circularly polarized systems is variable and need further correction for the average phase difference between the times of observations of the phase reference source and of 3C 286. This procedure is incompletely constrained as the relative phase of the two systems for the reference antenna is unknown. This correction from the difference in the RCP and LCP phases was determined from the delay and rate calibration. Subsequent phase calibration forced the rightand left-handed phase corrections to be the same. This procedure should result in a calibration error of the polarization angle of less than $2^{\circ}$.

The data were calibrated using standard techniques and phase-only self-calibration using AIPS program SCMAP. Some further editing of poor data was also undertaken during this phase. Final images in Stokes $I, Q$ and $U$ were produced using IMAGR with robustness 1.5 for $3 \mathrm{C} 43$ and 0 for $3 \mathrm{C} 454$.

\section{3. $V L B A 18 \mathrm{~cm}$}

The VLBA observations took place on 13 November 2000 and used four $8 \mathrm{MHz}$ bands centered at $1.651(18.15 \mathrm{~cm}), 1.659$ $(18.07 \mathrm{~cm}), 1.667(17.90 \mathrm{~cm})$, and $1.675(17.89 \mathrm{~cm}) \mathrm{GHz}$. All 
10 VLBA antennas and one VLA antenna participated. Rightand left-hand circular polarizations were used and all four correlation products were retained. The data were correlated by the NRAO VLBA correlator in Socorro, NM, USA. All processing was done in the NRAO AIPS package following standard procedures as outlined in Cotton (1993). 3C 84 was used as a fringe finder and for determining instrumental polarization. Polarization angle calibration was based on BL Lac with an assumed intrinsic polarization angle of $64^{\circ}$ and a rotation measure of $64 \mathrm{rad} \mathrm{m}^{-2}$ as obtained from VLA monitoring. In order to reduce the bandwidth smearing, the data were only averaged to $2 \mathrm{MHz}$ bandwidth. Final phase calibration of $3 \mathrm{C} 43$ and $3 \mathrm{C} 454$ used standard self calibration techniques applied by AIPS program SCMAP.

In order to determine the Faraday rotation across the $32 \mathrm{MHz}$ bandpass observed, each $8 \mathrm{MHz}$ band was imaged independently in Stokes $Q$ and $U$. Standard AIPS programs for determining rotation measure from a set of polarization images were inadequate, so a new program was written for the purpose. As a check for very large values of the rotation measure, each source was also imaged in Stokes $Q$ and $U$ in the individual $2 \mathrm{MHz}$ channels.

Since at $18 \mathrm{~cm}$ wavelength the pointing of the antennas should be quite good, it is possible to measure circular polarization. No amplitude self calibration was applied to the data before the final calibration. The initial calibration resulted in less than $1 \%$ instrumental circular polarization. To remove the remaining calibration error, the time and source averaged amplitude calibration from 3C 84, J0555+398 and BL Lac was determined from the amplitude self calibration of these calibrator sources. Imaging of J0555+398 and BL Lac suggest the remaining instrumental polarization was less that $0.03 \%$. 3C 43 and 3C 454 were imaged in all four Stokes parameters.

\section{Results}

\subsection{VLA $2 \mathrm{~cm}$}

The images derived from the VLA $2 \mathrm{~cm}$ measurements are shown in Fig. 2. In 3C 43 (Fig. 2, left), the jet is diverted from SSE to ENE. The polarization $E$-vectors are orthogonal to the line of the jet around the bend; suggesting that Faraday rotation is not important at this wavelength and that the dominant magnetic field component in the jet is longitudinal. 3C 454, shown in Fig. 2 (right), is not as resolved as 3C 43 by the VLA, but follows a similar pattern; the polarization vectors are orthogonal to the initial direction of the jet; however, the polarized intensity is low in the region where the jet bends to the west, possibly due to beam depolarization.

\subsection{MERLIN $6 \mathrm{~cm}$}

The MERLIN images of 3C 43 and 3C 454 are shown in Fig. 11 and have somewhat higher resolution than the VLA $2 \mathrm{~cm}$ images. As in the $2 \mathrm{~cm}$ images, the polarization $E$-vectors are generally orthogonal to the direction of the jet. Not shown in the $3 \mathrm{C} 43$ figure is a very weak extended feature near the northern component seen in Fig. 2, left.

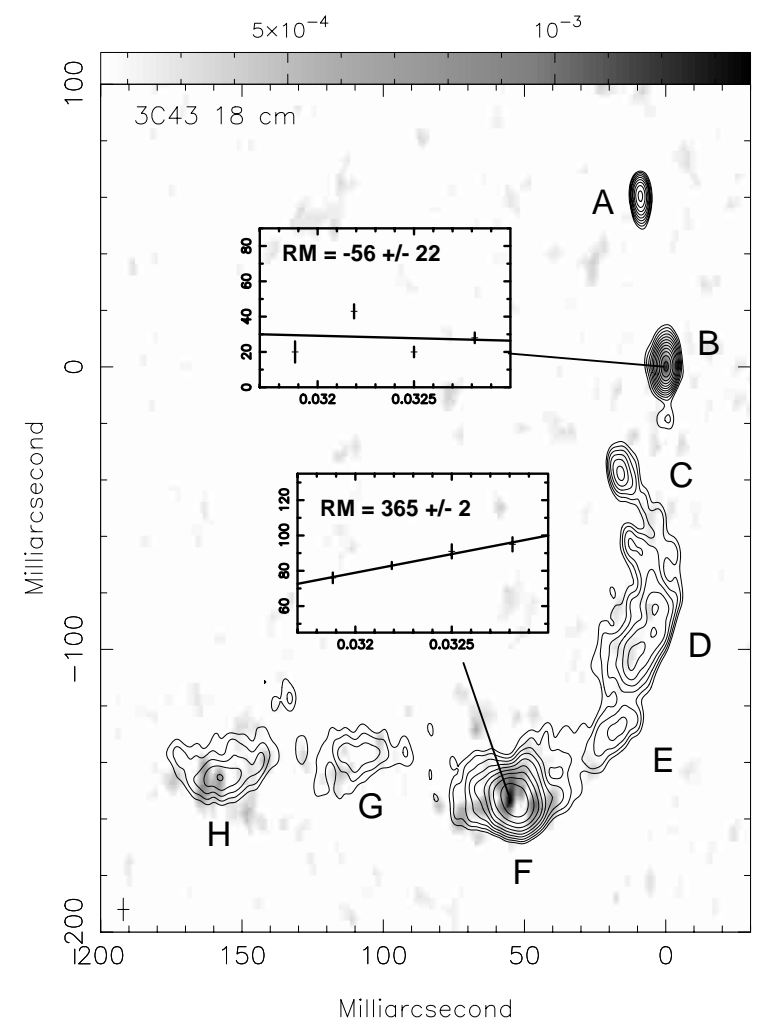

Fig. 1. Contour plot of the total intensity VLBA image of 3C 43 at $18 \mathrm{~cm}$ overlaid on the image of the polarized intensity. The lowest contour level is $2.5 \mathrm{mJy} /$ beam and the levels increase by $\sqrt{2}$. The wedge at the top indicates the polarized intensity scale. Superimposed plots show the measured values of the observed polarization angle for selected pixels as a function of $\lambda(m)^{2}$ with a line representing the fitted rotation measure. The RMS off-source noise level in the Stokes $I$ image is $359 \mu \mathrm{Jy}$. The RMS off-source noise levels in the Stokes $Q$ and $U$ images are 158,172,141 and $131 \mu \mathrm{Jy}$ for the four wavelength bands. Letters indicate component designations used in the text. The value of the rotation measure quoted is in the observer's frame $(\times 6.1$ in the rest frame of the source). The cross in the lower left corner indicates the size of the restoring beam $\left(8 \times 5\right.$ mas at $\left.\mathrm{PA}=0^{\circ}\right)$.

\section{3. $V L B A 18 \mathrm{~cm}$}

The $18 \mathrm{~cm}$ wavelength VLBA images of 3C 43 are shown in Figs. 1-5. Figure 1 gives the total intensity contours overlaid on the image of the polarized intensity (no bias correction applied). In addition, superimposed plots show the polarization angle against wavelength squared at two positions where the polarized intensity was highest. Figure 3 shows the total intensity contours overlaid on an image of the rotation measure derived from the four $8 \mathrm{MHz}$ bands observed. The rotation measure image is clipped below an SNR of 2.5 in the $8 \mathrm{MHz}$ band images. In Component B, south of the supposed core, Component A (see Sect. 4.1), the rotation measure is close to zero whereas the polarized region of the bend has a quite high rotation measure. Figure 4 shows an enlargement of the region of the bend in the jet in Fig. 3. If the Faraday rotation occurs in the rest frame of the source, then the wavelengths used to compute the rotation measure should be the emitted wavelengths; this increases the rotation measure by a factor of $(1+z)^{2}$. 

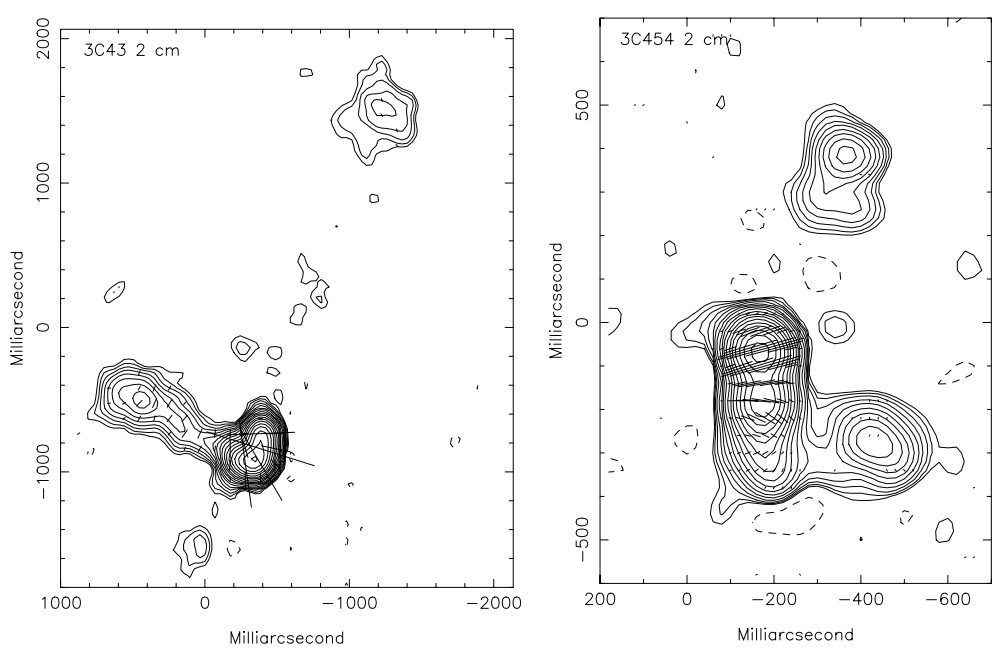

Fig. 2. Left: contour plot of the total intensity of $3 \mathrm{C} 43$ at $2 \mathrm{~cm}$ wavelength from the VLA observations with "robust $=0$ " weighting. Vectors are shown with lengths proportional to polarized intensity and with the orientation of the $E$-vectors. The feature near location (100, -1500$)$ is likely an imaging artifact. The lowest contour level is $0.5 \mathrm{mJy} / \mathrm{beam}$ and the levels increase by $\sqrt{2}$. The restoring beam size used was $139 \times 133 \mathrm{mas}$ at position angle $-20^{\circ}$. The RMS off-source noise level is $158 \mu \mathrm{Jy}$. Right: contour plot of the total intensity of $3 \mathrm{C} 454$ at $2 \mathrm{~cm}$ wavelength from the VLA observations imaged with uniform weighting. Minimum contour level is $0.4 \mathrm{mJy} / \mathrm{beam}$ and the levels increase by $\sqrt{2}$, the restoring beam size used was 80 mas. The RMS off-source noise level is $166 \mu \mathrm{Jy}$.

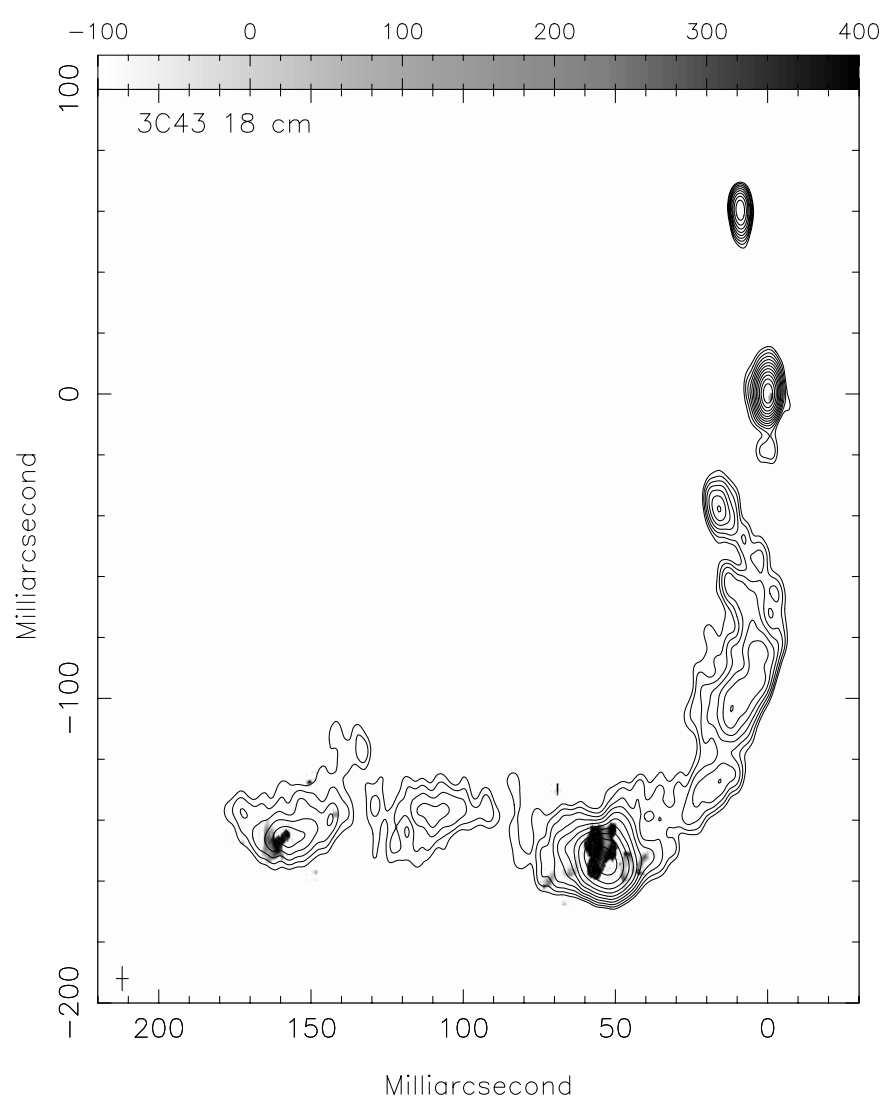

Fig. 3. Contour plot of the total intensity VLBA image of 3C 43 at $18 \mathrm{~cm}$ superimposed on the image of the rotation measure in the observer's frame $(\times 6.1$ in the source frame). The lowest contour level is $2 \mathrm{mJy} /$ beam and the levels increase by $\sqrt{2}$. The wedge at the top indicates the rotation measure scale.

The linearly polarized image of 3C 43 was bias corrected using the AIPS task POLCO and clipped below an SNR of 2.

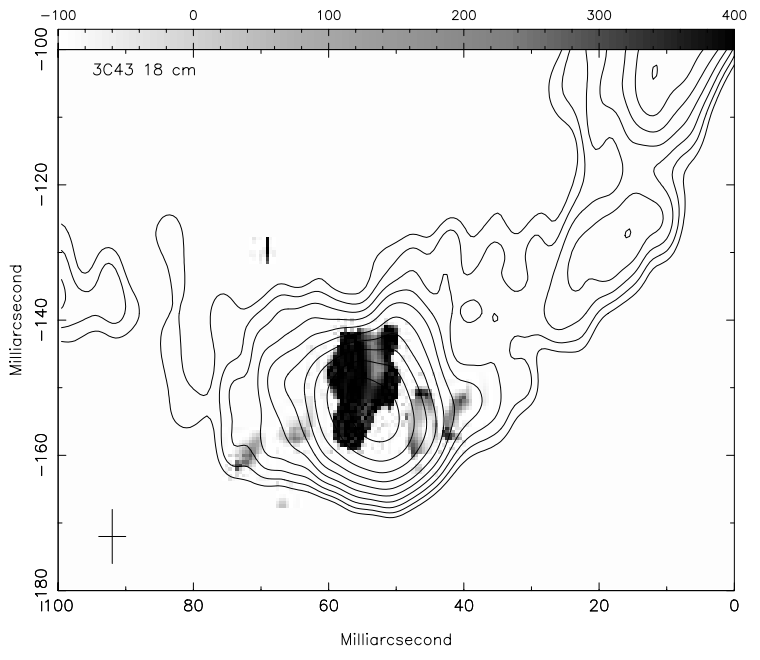

Fig. 4. Same as in Fig. 3 showing the region of the bend in the jet.

The fractional polarization is given in Fig. 5. Detections of polarized emission are weak, and it could not be detected over most of the observable jet above a level of $2 \%$. Prior to, and in the neighborhood of, the bend, the maximum fractional polarization is around $3 \%$; in the easternmost visible portion of the jet, the fractional polarization approaches $10 \%$.

The $18 \mathrm{~cm}$ wavelength VLBA images of $3 \mathrm{C} 454$ are shown in Figs. 6-8. Not shown is a weak, extended component detected in total intensity 200 mas west and 440 mas north of Component A (the presumed location of the core, see Sect. 4.2), placing it in the northern component seen in Fig. 2, right.

Figure 6 gives the total intensity contours for 3C 454 overlaid on the image of the polarized intensity (no bias correction applied). In addition, the superimposed plots show the polarization angle against wavelength squared at selected positions where the polarized intensity was highest. Figure 7 shows the 


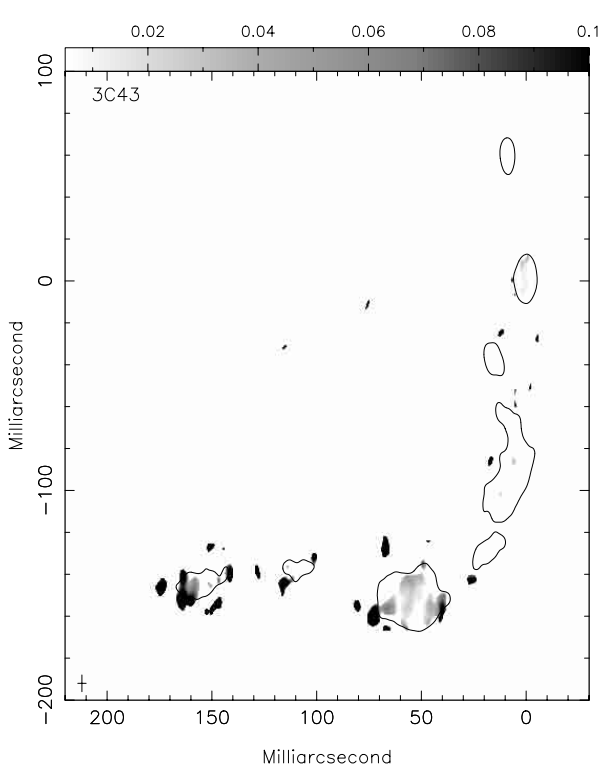

Fig. 5. The $5 \mathrm{mJy} /$ beam contour overlaid on the fractional polarization image of the VLBA $18 \mathrm{~cm}$ image of 3C 43. The wedge at the top indicates the fractional polarization scale.

total intensity contours overlaid on an image of the rotation measure derived from the four $8 \mathrm{MHz}$ band observed. The rotation measure image is clipped below a SNR of 2.5 in the $8 \mathrm{MHz}$ band images. The rotation measure is relatively low near the core, which is possibly located near the northern end of component $\mathrm{A}$, and increases to a moderately high level before decreasing again in the southernmost knot where the jet bends sharply to the west. The fractional polarization is given in Fig. 8 for which the linearly polarized image was bias corrected using the AIPS task POLCO and clipped below an SNR of 2. The fractional polarization is quite high, approaching $40 \%$ in places. Contributing to the high apparent polarization in the low surface brightness areas shown in Fig. 8 is an imaging artifact; polarized emission has more fine scale structure due to changes in Faraday rotation than does the total intensity making it less prone to brightness loss due to resolution than total intensity. This increases the apparent fractional polarization when the total intensity is more highly resolved than the linearly polarized intensity.

Integrated observed quantities for the regions marked in Figs. 1 and 6 are given in Table 1. The first column is the source and region identifier, the second column is the area of the region in synthesized beams, the third column is the integrated total intensity, the fourth column is the average fractional linear polarization, the fifth column is the average position angle of the electric vector, the sixth column is the average observed rotation measure, and the last column is the average rotation measure derived in the frame of the source. Estimates of the error in the rotation measure, derived from signal-to-noise considerations, are given in parentheses following the rotation measure. The systematic error in the rotation measure is dominated by the unknown error in the assumed rotation measure calibrator, $\mathrm{BL} \mathrm{Lac}$, and is probably smaller than a few $\mathrm{rad} \mathrm{m}^{-2}$.

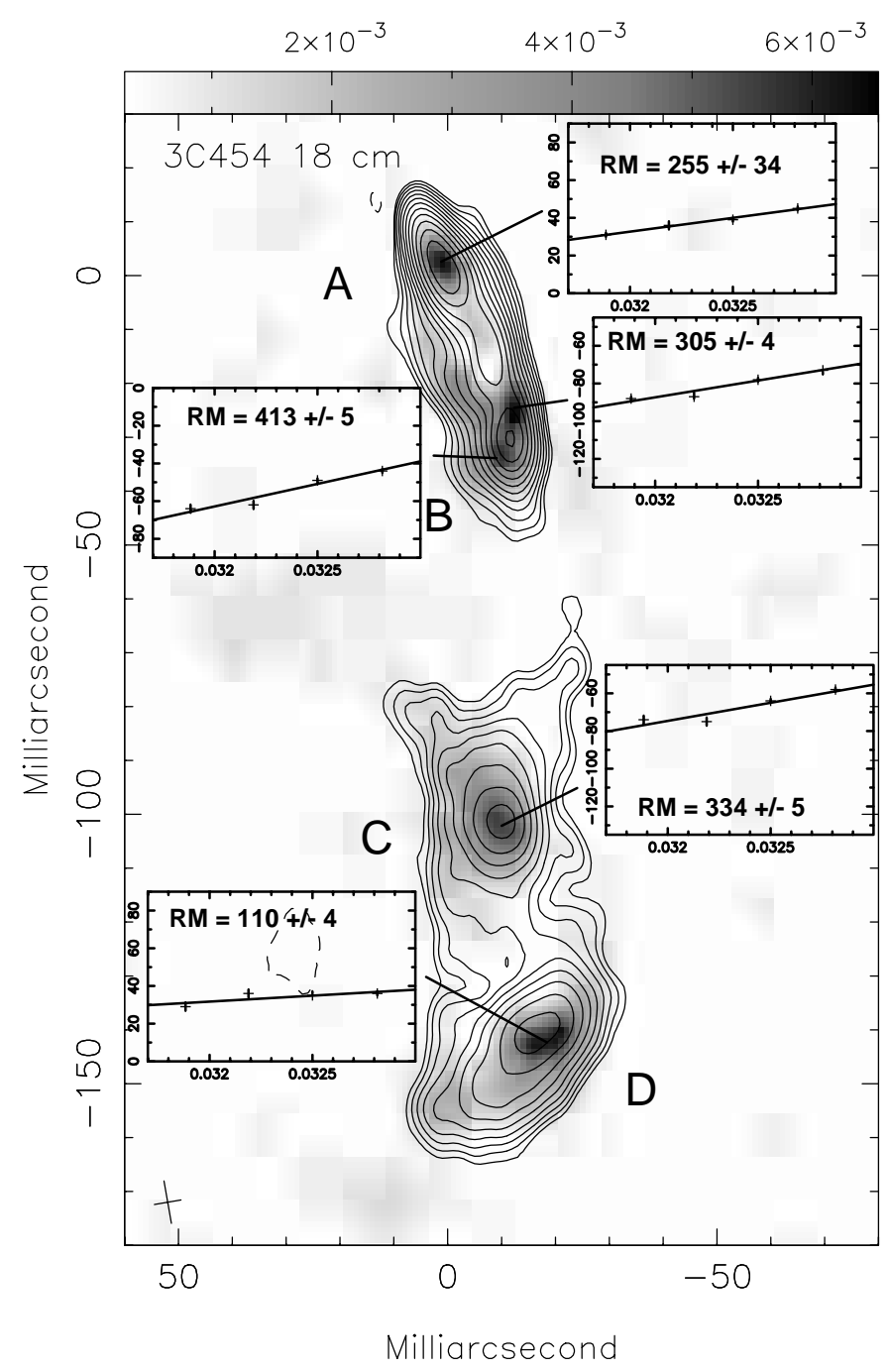

Fig. 6. Contour plot of the total intensity VLBA image of 3C 454 at $18 \mathrm{~cm}$ overlaid on the image of the polarized intensity. The lowest contour level is $1.5 \mathrm{mJy} / \mathrm{beam}$ and the levels increase by $\sqrt{2}$. The wedge at the top indicates the polarized intensity scale. Superimposed plots show the measured values of the observed polarization angle for selected pixels as a function of $\lambda(m)^{2}$ with a line representing the fitted rotation measure. The value of the rotation measure quoted is in the observer's frame ( $\times 7.6$ in the rest frame of the source). The RMS off-source noise level in the Stokes $I$ image is $314 \mu \mathrm{Jy}$. The RMS offsource noise levels in the Stokes $Q$ and $U$ images are 270, 300, 210 and $156 \mu \mathrm{Jy}$ for the four wavelength bands. Letters indicate component designations used in the text. The cross in the lower left corner indicates the size of the restoring beam $\left(8 \times 5\right.$ mas at $\left.\mathrm{PA}=10^{\circ}\right)$.

\subsection{Circular polarization at $18 \mathrm{~cm}$}

Images of the calibrators J0555+398 and BL Lac show no circular polarization above the three sigma level, $0.04 \%$ and $0.03 \%$ respectively. $3 \mathrm{C} 84$ shows possible polarized emission at the $-0.06 \%$ level. However, circular polarization has been reported in 3C 84 (Homan \& Wardle 1999). The circular polarization image of $3 \mathrm{C} 454$ at $18 \mathrm{~cm}$ is shown in Fig. 9. The bright negative region on the south-west (trailing) end of the brightest region of the jet has a peak of $-0.7 \%$ and is 4.4 times the RMS off-source noise level of $69 \mu \mathrm{Jy} /$ beam. The Stokes $V$ 
Table $1.18 \mathrm{~cm}$ region averaged quantities.

\begin{tabular}{lcccccc}
\hline \hline Region & $\begin{array}{c}\text { Area } \\
(\text { beams })\end{array}$ & $\begin{array}{c}S \\
(\mathrm{mJy})\end{array}$ & $\begin{array}{c}m \\
(\%)\end{array}$ & $\begin{array}{c}E V P A \\
\left({ }^{\circ}\right)\end{array}$ & $\begin{array}{c}R M \\
\left(\mathrm{rad} \mathrm{m}^{-2}\right)\end{array}$ & $\begin{array}{c}R M \text { (source) } \\
\left(\mathrm{rad} \mathrm{m}^{-2}\right)\end{array}$ \\
\hline 3C 43 A & 7.3 & 56.2 & 0.3 & -58 & & \\
3C 43 B & 7.4 & 181.6 & 0.7 & 45 & $-360(10)$ & $-2178(60)$ \\
3C 43 C & 6.0 & 36.9 & 0.8 & 45 & & \\
3C 43 D & 53.6 & 256.1 & 1.3 & 76 & $625(18)$ & $3781(109)$ \\
3C 43 E & 22.3 & 66.6 & 0.4 & 23 & $2353(14)$ & $14236(85)$ \\
3C 43 F & 32.3 & 418.1 & 1.3 & 78 & $507(6)$ & $3067(36)$ \\
3C 43 G & 32.3 & 73.8 & 1.2 & 63 & $-312(23)$ & $-1888(140)$ \\
3C 43 H & 24.3 & 95.7 & 2.8 & -73 & $155(13)$ & $938(79)$ \\
3C 454 A & 11.1 & 242.4 & 3.9 & 34 & $148(8)$ & $1194(65)$ \\
3C 454 B & 12.4 & 211.9 & 10.9 & -66 & $661(5)$ & $5334(40)$ \\
3C 454 C & 32.8 & 194.4 & 21.3 & -71 & $327(4)$ & $2639(32)$ \\
3C 454 D & 28.6 & 211.4 & 16.6 & 47 & $211(3)$ & $1703(24)$ \\
\hline
\end{tabular}

${ }^{1}$ Region locations are indicated in Fig. 1.

${ }^{2}$ Region locations are indicated in Fig. 6.

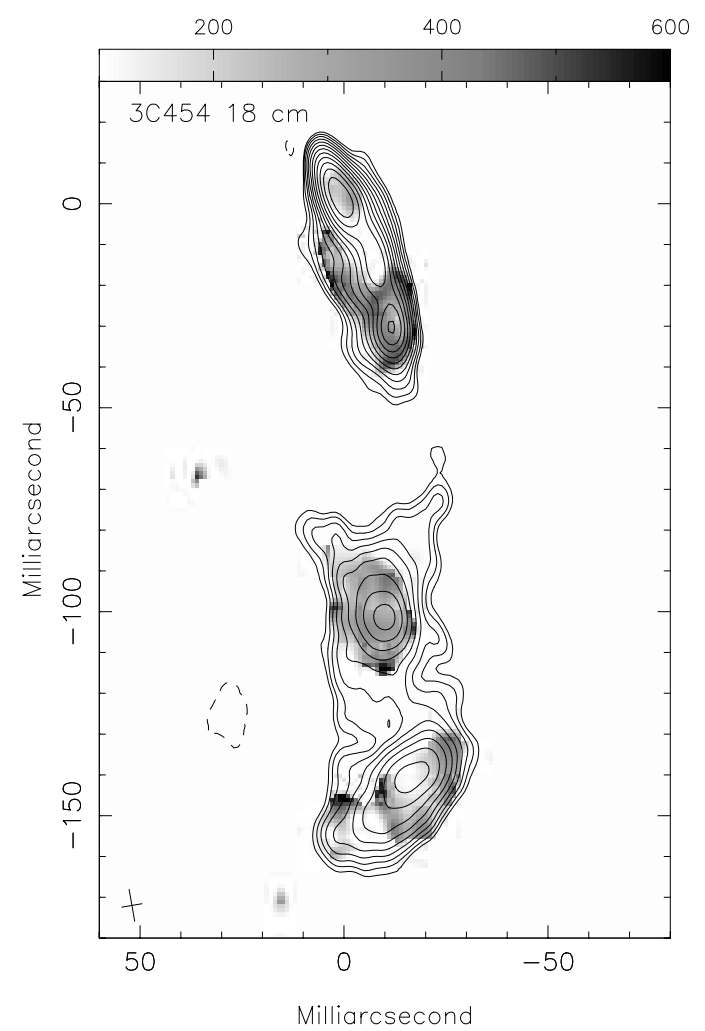

Fig. 7. Contour plot of the total intensity VLBA image of 3C 454 at $18 \mathrm{~cm}$ superimposed on image of the rotation measure in the observer's frame ( $\times 7.6$ in the source frame). The lowest contour level is $1.5 \mathrm{mJy} / \mathrm{beam}$ and the levels increase by $\sqrt{2}$. The wedge at the top indicates the rotation measure scale.

image of 3C 43 shows no circularly polarized emission above the RMS noise level of $80 \mu \mathrm{Jy} /$ beam.

\subsection{Comparison of VLA and MERLIN results}

The MERLIN $6 \mathrm{~cm}$ images have somewhat better resolution than the VLA $2 \mathrm{~cm}$ images. To make proper comparison

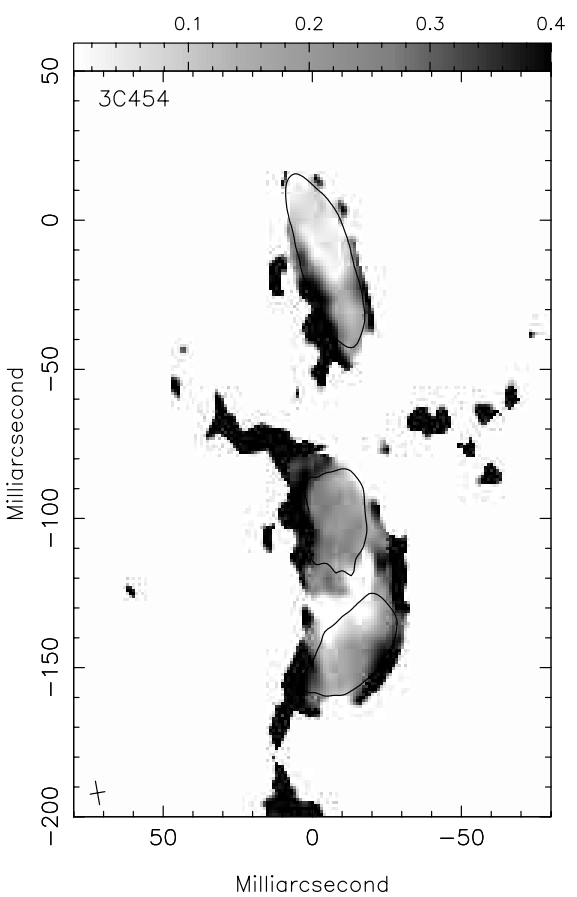

Fig. 8. The $5 \mathrm{mJy} /$ beam contour overlaid on the fractional polarization image of the VLBA $18 \mathrm{~cm}$ image of $3 \mathrm{C} 454$. The wedge at the top indicates the fractional polarization scale.

between the two frequencies, the MERLIN images were convolved to the 80 mas restoring beam size used for the uniform weighted VLA images. The two sets of images were both phase referenced to (different) astrometric calibrators but features appeared misaligned at the 50 mas level. The two 6 and $2 \mathrm{~cm}$ image pairs were aligned using the brightest component in each source; this procedure appears to align the sources adequately.

A comparison of the images of $3 \mathrm{C} 43$ is shown in Fig. 12 and 3C 454 in Fig. 13. The properties of the resultant images at selected pixels are given in Table 2 ; the locations of the pixels are indicated in Figs. 12 and 13. This table gives the 
Table 2. 2-6 cm comparison.

\begin{tabular}{|c|c|c|c|c|c|c|c|c|c|}
\hline \multirow[b]{2}{*}{ Pixel } & \multicolumn{3}{|c|}{$2 \mathrm{~cm}$} & \multicolumn{3}{|c|}{$\overline{6 \mathrm{~cm}}$} & \multirow[b]{2}{*}{$\begin{array}{l}R M \\
\left(\operatorname{rad~m}^{-2}\right)\end{array}$} & \multirow[b]{2}{*}{$\begin{array}{r}R M(\text { source }) \\
\quad\left(\operatorname{rad~m^{-2})}\right.\end{array}$} & \multirow[b]{2}{*}{$\alpha$} \\
\hline & $\begin{array}{r}S \\
\left(\mathrm{mJy} \mathrm{bm}^{-1}\right)\end{array}$ & $\begin{array}{r}m \\
(\%)\end{array}$ & $\begin{array}{c}E V P A \\
\left({ }^{\circ}\right)\end{array}$ & $\begin{array}{r}S \\
\left(\mathrm{mJy} \mathrm{bm}^{-1}\right)\end{array}$ & $\begin{array}{r}m \\
(\%)\end{array}$ & $\begin{array}{c}E V P A \\
\quad\left({ }^{\circ}\right)\end{array}$ & & & \\
\hline $3 \mathrm{C} 43 \mathrm{a}^{1}$ & 83.6 & 7.7 & $-86(0.7)$ & 144.8 & 5.9 & $-70.1(1.3)$ & $87(14)$ & $536(86)$ & $-0.50(0.02)$ \\
\hline $3 \mathrm{C} 43 \mathrm{~b}$ & 137.2 & 6.2 & $16(0.5)$ & 294.1 & 5.3 & $9.6(0.6)$ & $-35(12)$ & $-212(74)$ & $-0.70(0.01)$ \\
\hline $3 \mathrm{C} 43 \mathrm{c}$ & 41.2 & 3.2 & $-11(3.5)$ & 80.9 & 2.3 & $-24(5)$. & $-69(46)$ & $-417(280)$ & $-0.62(0.03)$ \\
\hline $3 \mathrm{C} 43 \mathrm{~d}$ & 3.7 & 22. & $-30(5.7)$ & 13.7 & 29. & -35 (14.) & $-27(81)$ & $-163(500)$ & $-1.20(0.25)$ \\
\hline $3 \mathrm{C} 454 \mathrm{a}^{2}$ & 124.3 & 18.7 & $-74.2(0.2)$ & 272.9 & 18.2 & $-84.7(0.1)$ & $-57.3(10)$ & $-435(76)$ & $-0.72(.002)$ \\
\hline $3 \mathrm{C} 454 \mathrm{~b}$ & 72.6 & 10.5 & $83.2(0.6)$ & 180.0 & 10.9 & $64.6(0.4)$ & $-101.4(311)$ & $-770(84)$ & $-0.83(.004)$ \\
\hline $3 \mathrm{C} 454 \mathrm{c}$ & 9.4 & 13.8 & $-48.3(3.3)$ & 17.1 & 21.6 & $-57.9(1.6)$ & $-52.3(21)$ & $-397(159)$ & $-0.55(.036)$ \\
\hline $3 \mathrm{C} 454 \mathrm{~d}$ & 9.0 & 5.2 & $-33(8)$ & 19.3 & 4.8 & $-27(11)$ & $33(74)$ & $251(569)$ & $-0.69(0.04)$ \\
\hline
\end{tabular}

${ }^{1}$ Pixel locations are indicated in Fig. 12.

${ }^{2}$ Pixel locations are indicated in Fig. 13.

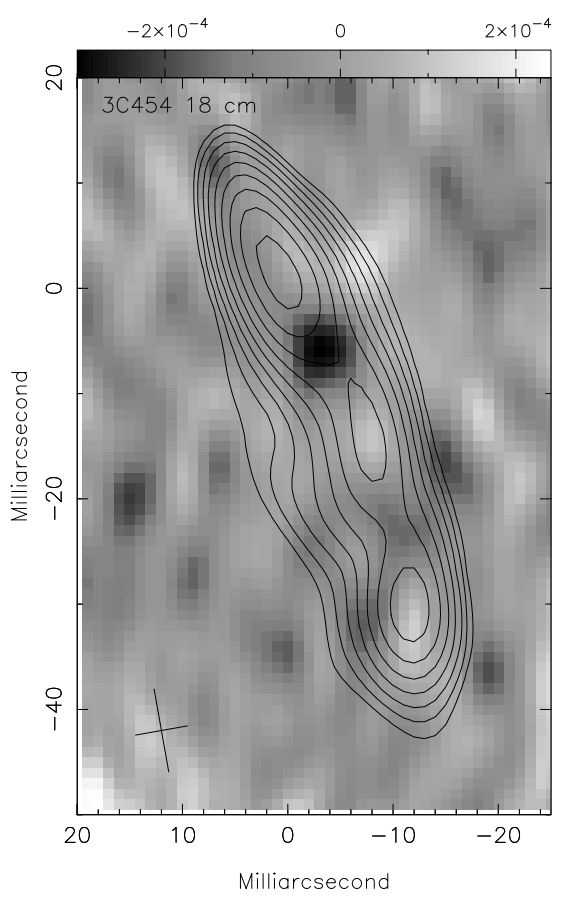

Fig. 9. Total intensity contours overlaid on a Stokes $V$ image of the inner portion of the VLBA $18 \mathrm{~cm}$ image of $3 \mathrm{C} 454$. The lowest contour is $5 \mathrm{mJy} /$ beam and the levels increase by $\sqrt{2}$. The wedge at the top indicates the fractional polarization scale.

total intensity and linearly polarized fraction and position angle at 2 and $6 \mathrm{~cm}$, the derived rotation measure $(R M)$ as observed and in the source rest frame, and the spectral index. The SNR of the polarized images at most of the pixels sampled is sufficiently large that bias corrections are negligible; such corrections were made (AIPS program POLCO) when needed. Error estimates derived from signal-to-noise considerations are given in parentheses behind the estimated value, except for the rotation measure columns for which the uncertainties include a $2^{\circ}$ systematic uncertainty in the MERLIN calibration. Note: that this systematic error (10 $\mathrm{rad} \mathrm{m}^{-2}$ assumed) will be common to all RMs derived for a each source and should not be included in a comparison of different regions. Even though the

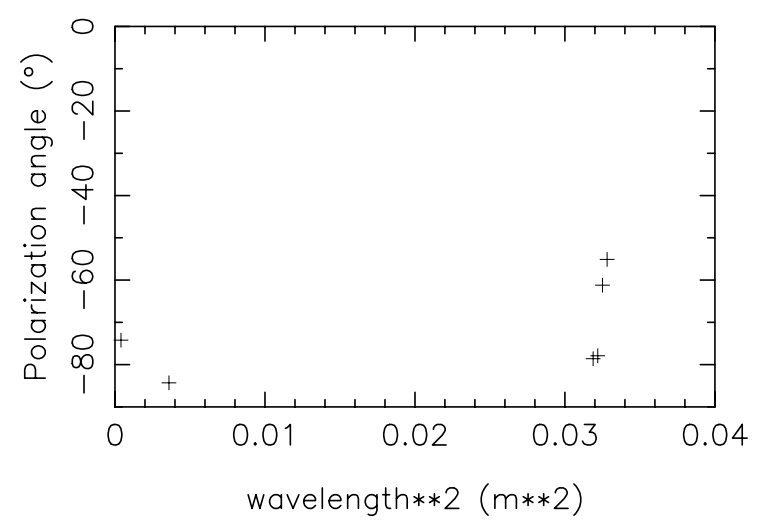

Fig. 10. The measured polarization vectors for the integrated northern end of the jet in 3C 454 ("a" in Fig. 13 and "A" + "B" in Fig. 6) against wavelength squared. The formal error bars are smaller than the symbols plotted

resolutions are the same in the pairs of images, the surface brightness sensitivities are not; the spectral index estimates for the more extended components will be biased in the positive direction by the better short baseline coverage of the VLA at $2 \mathrm{~cm}$. The rotation measure estimated may also be affected, but in a less predictable manner.

The results of fitting Gaussians to the full resolution MERLIN $6 \mathrm{~cm}$ and VLA $2 \mathrm{~cm}$ images of 3C 454 are shown in Table 3. This table gives the fitted flux densities, positions relative to Component a and the deconvolved Gaussian component parameters. Formal errors are given in parentheses behind each value.

\section{Discussion}

\section{1. $3 C 43$}

The location of the core in $3 \mathrm{C} 43$ is unclear from our observations. It is possibly component A in Fig. 1 but there is no comparable resolution shorter wavelength data to verify a flat or inverted spectrum. The shorter wavelength, lower resolution data shown in Fig. 12 and summarized in Table 2 show that this area of the source has the flattest spectrum. 
Table 3. 2 and $6 \mathrm{~cm}$ model fit for 3C 454 .

\begin{tabular}{|c|c|c|c|c|c|c|c|}
\hline Comp & $\begin{array}{c}\lambda \\
(\mathrm{cm})\end{array}$ & $\begin{array}{c}S \\
(\mathrm{mJy})\end{array}$ & $\begin{array}{r}\Delta \alpha \\
(\mathrm{mas})\end{array}$ & $\begin{array}{r}\Delta \delta \\
\text { (mas) }\end{array}$ & $\begin{array}{r}\text { Maj } \\
(\mathrm{mas})\end{array}$ & $\begin{array}{r}\text { Min } \\
\text { (mas) }\end{array}$ & $\begin{array}{l}\text { PA } \\
\left({ }^{\circ}\right)\end{array}$ \\
\hline \multirow[t]{2}{*}{$3 C 454 a^{1}$} & 2 & $140.9(0.3)$ & $=0$ & $=0$ & $36.1(1.0)$ & $21.9(0.1)$ & $22.2(0.7)$ \\
\hline & 6 & $294.1(1.0)$ & $=0$ & $=0$ & $36.6(0.2)$ & $10.1(0.1)$ & $26.8(0.2)$ \\
\hline \multirow[t]{2}{*}{$3 \mathrm{C} 454 \mathrm{~b}$} & 2 & $86.7(0.3)$ & $-6.32(0.09)$ & $-113.74(0.11)$ & $54.4(0.4)$ & $20.2(0.6)$ & $-4.3(0.5)$ \\
\hline & 6 & $257.1(0.8)$ & $-6.40(0.04)$ & $-114.0(0.11)$ & $78.3(0.2)$ & $19.6(0.3)$ & $4.9(0.1)$ \\
\hline \multirow[t]{2}{*}{$3 \mathrm{C} 454 \mathrm{c}$} & 2 & $28.4(0.4)$ & $-11.28(0.48)$ & $-221.56(0.65)$ & $114.6(1.5)$ & $52.8(1.5)$ & $17.4(0.8)$ \\
\hline & 6 & $45.5(1.1)$ & $-0.20(0.57)$ & $-223.9(0.9)$ & $128.3(2.0)$ & $27.4(1.0)$ & $21.3(0.5)$ \\
\hline \multirow[t]{2}{*}{$3 \mathrm{C} 454 \mathrm{~d}$} & 2 & $19.3(0.5)$ & $-264.16(0.96)$ & $-210.68(0.84)$ & $111.1(2.0)$ & $67.1(2.0)$ & $60.4(1.8)$ \\
\hline & 6 & $46.0(1.2)$ & $-265.4(0.9)$ & $-212.3(1.0)$ & $116.8(2.0)$ & $53.5(2.0)$ & $47.6(0.3)$ \\
\hline
\end{tabular}

${ }^{1}$ Pixel locations are indicated in Fig. 13.
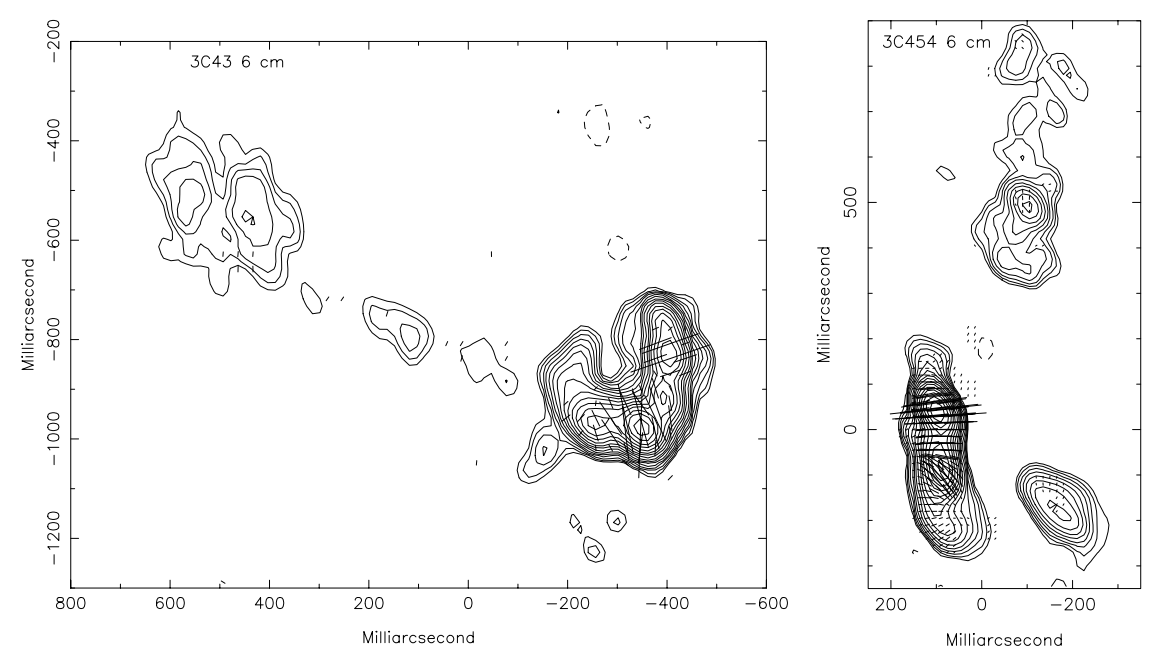

Fig. 11. Left: contour plot of the total intensity MERLIN image of 3C 43 at $6 \mathrm{~cm}$ with polarized (E-vector) emission vectors. The lowest contour level is $1.0 \mathrm{mJy} / \mathrm{beam}$ and the levels increase by $\sqrt{2}$. The RMS off-source noise level is $257 \mu \mathrm{Jy}$. The restoring beam is $59.4 \times 40.7 \mathrm{mas}$ at position angle $30^{\circ}$. Right: contour plot of the total intensity MERLIN image of 3C 454 at $6 \mathrm{~cm}$ with polarized (E-vector) emission vectors. The lowest contour level is $1.0 \mathrm{mJy} /$ beam and the levels increase by $\sqrt{2}$. The RMS off-source noise level is $237 \mu \mathrm{Jy}$. The restoring beam is $62.5 \times 41.3$ mas at position angle $27^{\circ}$.

Fanti et al. (2002) point out that the low frequency spectrum (between 0.4 and $5 \mathrm{GHz}$ ) of $\mathrm{A}$ is markedly inverted, with some evidence (Mantovani et al. 2002; Mantovani et al. 2003) that the spectrum peaks between 5 and $8.4 \mathrm{GHz}$. We will therefore assume that the core is at or near component $\mathrm{A}$ in Fig. 1 and that the main jet is initially to the south and after 210 mas $(1.9 \mathrm{kpc})$ bends to the east where it continues at least $1.1^{\prime \prime}(10.0 \mathrm{kpc})$. A faint feature 2.6" $(24 \mathrm{kpc})$ to the north in Fig. 2 is presumed to be the counter lobe.

The region of the bend in the jet is accompanied by a bright knot (component $\mathrm{F}$ in Fig. 1, component $\mathrm{b}$ in Fig. 12). The source is relatively weakly polarized, especially at lower frequencies (see Fig. 1, Tables 1 and 2). There are only two relatively bright positions in the $18 \mathrm{~cm}$ polarization emission, one (component B) relatively close to the core has fairly low rotation measure while in component $\mathrm{F}$, large rotation measure is seen. This is supported in Table 1 where regions D, E, and F (in the area of the bend) all show large integrated rotation measures.

The lower resolution, shorter wavelength data summarized in Table 2 show only relatively modest rotation measure in the area of the bend (component b). This shorter wavelength data has much better surface brightness sensitivity than the $18 \mathrm{~cm}$ VLBA data and may well be dominated by more extended emission.

The patchy nature of the $18 \mathrm{~cm}$ polarization, as well as the fine scale structure seen in the Faraday rotation in $3 \mathrm{C} 43$ seen in Fig. 3 (details in Fig. 4), suggest that the jet is being viewed through a thick Faraday screen with occasional holes letting linearly polarized emission through. The low fractional polarization of the shorter wavelength data is in agreement with this possibility. This strongly suggests that the region of the bend in the jet is being viewed through a dense thermal plasma. This plasma could be the result of the jet ionizing the interstellar medium.

\section{2. $3 C 454$}

The location of the core in 3C 454 cannot be established with the present data but it is plausibly near the northern end of component A in Fig. 6. This area is less polarized than the rest of the source which could indicate the region of the core. The main jet 

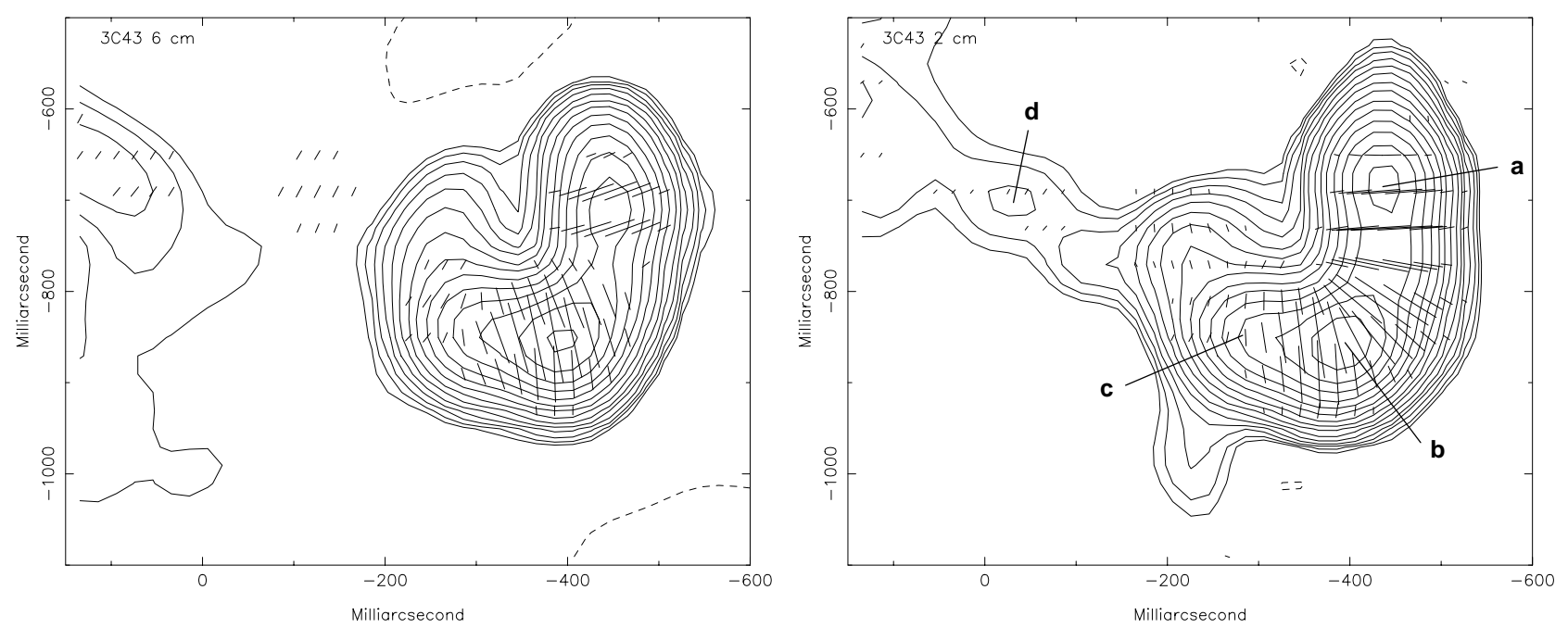

Fig. 12. Left: contour plot of the total intensity MERLIN $6 \mathrm{~cm}$ image of $3 \mathrm{C} 43$ at 80 mas resolution with polarized (E-vector) emission vectors. The lowest contour level is $3.0 \mathrm{mJy} / \mathrm{beam}$ and the levels increase by $\sqrt{2}$. The RMS off-source noise level is $1973 \mu \mathrm{Jy}$. Right: contour plot of the total intensity $2 \mathrm{~cm}$ VLA image of $3 \mathrm{C} 43$ at 80 mas resolution with polarized ( $E$-vector) emission vectors. The lowest contour level is $0.8 \mathrm{mJy} / \mathrm{beam}$ and the levels increase by $\sqrt{2}$. The RMS off-source noise level is $228 \mu \mathrm{Jy}$. Letters indicate locations described in the text.
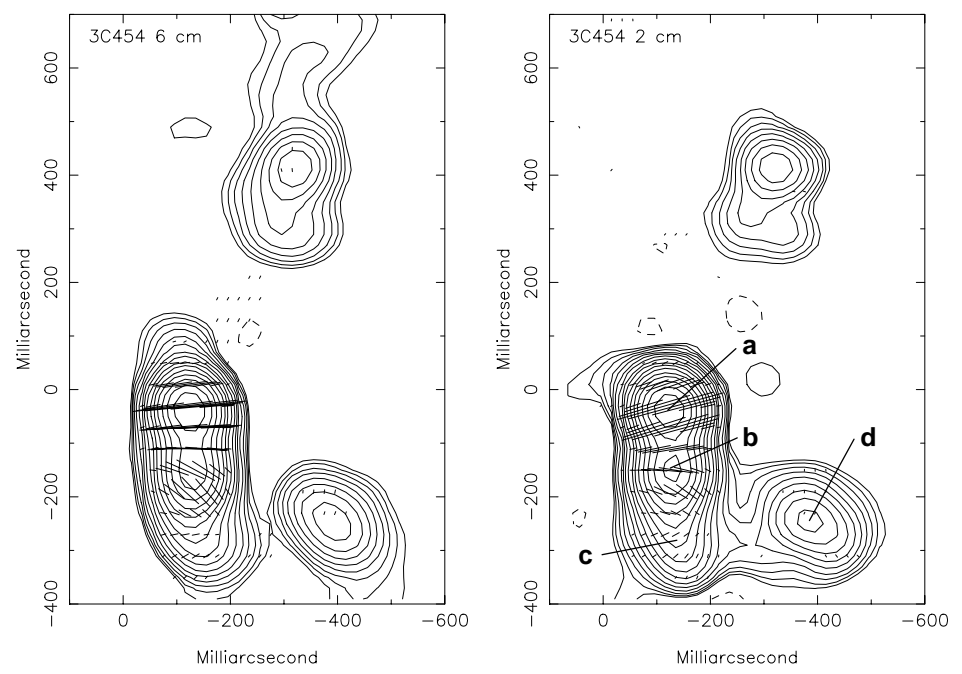

Fig. 13. Left: contour plot of the total intensity MERLIN $6 \mathrm{~cm}$ image of 3C 454 at 80 mas resolution with polarized (E-vector) emission vectors. The lowest contour level is $1.5 \mathrm{mJy} /$ beam and the levels increase by $\sqrt{2}$. The RMS off-source noise level is $540 \mu \mathrm{Jy}$. Right: contour plot of the total intensity $2 \mathrm{~cm}$ VLA image of $3 \mathrm{C} 454$ at 80 mas resolution with polarized ( $E$-vector) emission vectors. The lowest contour level is $0.5 \mathrm{mJy} / \mathrm{beam}$ and the levels increase by $\sqrt{2}$. The RMS off-source noise level is $166 \mu \mathrm{Jy}$. Letters indicate locations described in the text.

appears to continue to the south with several knots visible in the $18 \mathrm{~cm}$ image. The MERLIN $6 \mathrm{~cm}$ image (Fig. 11) indicates that the bend to the west occurs further south than the visible region at $18 \mathrm{~cm}$, at around 330 mas $(3.0 \mathrm{kpc})$ from the bright northern end of the visible jet. Both the MERLIN $6 \mathrm{~cm}$ and VLA $2 \mathrm{~cm}$ images (Fig. 2) show emission 220 mas ( $2.0 \mathrm{kpc}$ ) to the west, as well as presumed counter-jet emission 740 mas $(6.7 \mathrm{kpc})$ to the north.

The westward bend in the jet of 3C 454 does not appear to be the site of a brightening in the jet. This suggests that this bend is not the result of a jet-ISM interaction which should compress and shock the jet. Since this object is a quasar, the jet is presumably close to the line of sight and the bend in the angle of the jet could be the result of a hydrodynamic instability greatly magnified by the observing geometry.
The jet seen in Fig. 6 is different in another way; high rotation measures are seen over the entire visible jet (168 mas), a projected distance of $1.5 \mathrm{kpc}$. Moreover, the rotation measure shown in Fig. 6 and given in Table 1 is consistently of the opposite sign of that seen in the lower resolution, 2-6 cm values given in Table 2. The cause of this discrepancy could be due to the different resolutions of the long and short wavelength measurements. The short wavelength measurements, especially at $2 \mathrm{~cm}$, have much better short baseline coverage and are much more sensitive to extended emission.

On the other hand, a Gaussian fit (AIPS program JMFIT) to the MERLIN full resolution image (Table 3) shows component a (as defined in Fig. 13) to be approximately the size of the region labeled A \& B in Fig. 6. A rough estimate of the size of this region from Fig. 6 is $60 \times 13$ mas at position angle $20^{\circ}$. 
This is to be compared to the Gaussian fit to the full resolution MERLIN image (Fig. 11, right) of $33.5 \times 9.6$ mas at position angle $26.8^{\circ}$. The apparently smaller size at the lower resolution is due to the non-Gaussian shape of the structure.

The apparent agreement in the size of this inner region of the jet between the high and low resolution images suggests that the $18 \mathrm{~cm}$ image includes all of the flux density. An integrated measurement over the $\mathrm{A}+\mathrm{B}$ region of Fig. 6 gives a rotation measure of $502 \pm 8 \mathrm{rad} \mathrm{m}^{-2}$ (our frame); the comparable value at short wavelengths is $-54.6 \pm 1.2 \mathrm{rad} \mathrm{m}^{-2}$. The fractional linear polarization at $18 \mathrm{~cm}$ is also much smaller, $4.5 \%$, versus 18.2 and $18.7 \%$ at 6 and $2 \mathrm{~cm}$ wavelength.

The behaviour of the polarization angle with wavelength appears to be very different from a $\lambda^{2}$ law. The measured values for this region are shown in Fig. 10. This behavior can be explained by thermal plasma mixed with the relativistic gas (Burn 1966). The Faraday dispersion caused by such thermal plasma would depolarize the longer wavelength emission. At $18 \mathrm{~cm}$ the emission is dominated by the brighter, initial portion of the jet for which the fractional linear polarization is low, a few percent (see Fig. 8).

Such thermal plasma mixed with the relativistic gas could also result in conversion of Stokes $U$ to Stokes $V$ (Kennett \& Melrose 1998) and thus produce significant circular polarization. Such a feature is possibly seen in Fig. 9 where a $-0.7 \%$ feature is 4.4 times the off-source noise and far above the instrumental circular polarization level. This feature occurs just where the jet exhibits a drop in surface brightness; this is 15 mas $(200 \mathrm{pc})$ from the presumed location of the nucleus.

The Faraday rotation measured for 3C 43 could show a similar effect; the signs of the Faraday rotation shown at 18 and $6-2 \mathrm{~cm}$ in Tables 1 and 2 are opposite in sign and lower in magnitude at the shorter wavelengths. However, the weak polarization and great difference in resolution make this result suspect in the present data.

\section{Conclusions}

The region of the bend in the jet in $3 \mathrm{C} 43$ shows the expected large Faraday effects in the high resolution $18 \mathrm{~cm}$ image. The weak and patchy nature of the polarized emission and the fine scale variations of the Faraday rotation suggest that the source is being viewed through a thick Faraday screen which may be the result of jet-ISM interaction.

The jet in 3C 454 has quite different properties; there does not appear to be a brightening in the jet near the westward bend in the jet suggesting that it is not caused by a collision with a cloud in the ISM. However, the jet is relatively strongly polarized, 10-20\% at all wavelengths observed and the $18 \mathrm{~cm}$ data show large Faraday rotation all along the visible portion of the jet, $1.5 \mathrm{kpc}$ in projected length. Modeling of the MERLIN $6 \mathrm{~cm}$ results suggests that the $18 \mathrm{~cm}$ image includes all of the emission seen at shorter wavelengths so the differences in the observed Faraday rotation at long and short wavelengths are unlikely to be due to resolution effects. In particular, the sign of the measured Faraday rotation is opposite and much lower in magnitude at shorter wavelengths. This is in agreement with the model of Burn (1966) of the effects of thermal plasma mixed with the relativistic radiating particles. These are the conditions that should give rise to the conversion of linear to circular polarization (Jones \& Odell 1977). The likely detection of circular polarization in the jet in 3C 454 at $18 \mathrm{~cm}$ supports this interpretation. Thus, there is evidence that the jet in $3 \mathrm{C} 454$ contains entrained thermal material.

Acknowledgements. The authors would like to thank Dan Homan for suggestions in measuring and interpreting the circular polarization results and the referee, Chris Salter, for many helpful suggestions in the presentation of this work. The National Radio Astronomy Observatory is a facility of the National Science Foundation operated under cooperative agreement by Associated Universities, Inc. MERLIN is a UK National Facility operated by the University of Manchester on behalf of PPARC.

\section{References}

Akujor, C. E., Spencer, R. E., \& Saikia, D. J. 1991a, A\&A, 249, 337 Akujor, C. E., Spencer, R. E., Zhang, F. J., et al. 1991b, MNRAS, 250, 215

Axon, D. J., Capetti, A., Fanti, R., et al. 2000, AJ, 120, 2284

Burn, B. J. 1966, MNRAS, 133, 67

Cotton, W. D. 1993, AJ, 106, 1241

Cotton, W. D., Dallacasa, D., Fanti, C., et al. 1997, A\&A, 325, 493

de Vries, W. H., O’Dea, C. P., Baum, S. A., \& Barthel, P. D. 1999, ApJ, 526, 27

Fanti, C., Fanti, R., Dallacasa, D., et al. 2002, A\&A, 396, 801

Fanti, C., Fanti, R., Dallacasa, D., et al. 1995, A\&A, 302, 317

Fanti, R., Fanti, C., Schilizzi, R., et al. 1990, A\&A, 231, 33

Gelderman, R., \& Whittle, M. 1994, ApJS, 91, 491

Homan, D. C., \& Wardle, J. F. C. 1999, AJ, 118, 1942

Inoue, M., Tabara, H., Kato, T., \& Aizu, K. 1995, PASJ, 47, 725

Jones, T. W., \& Odell, S. L. 1977, ApJ, 214, 522

Junor, W., Salter, C. J., Saikia, D. J., Mantovani, F., \& Peck, A. B. 1999, MNRAS, 308, 955

Kapahi, V. K. 1981, A\&AS, 43, 381

Kennett, M., \& Melrose, D. 1998, Publ. Astron. Soc. Austr., 15, 211

Lehnert, M. D., Miley, G. K., Sparks, W. B., et al. 1999, ApJS, 123, 351

Lüdke, E., Garrington, S. T., Spencer, R. E., et al. 1998, MNRAS, 299, 467

Mantovani, F., Junor, W., Bondi, M., et al. 1998, A\&A, 332, 10

Mantovani, F., Junor, W., Ricci, R., et al. 2002, A\&A, 389, 58

Mantovani, F., Bondi, M., Saikia, D. J., et al. 2003, PASA, 20, 85

Murgia, M., Fanti, C., Fanti, R., et al. 1999, A\&A, 345, 769

O’Dea, C. P. 1998, PASP, 110, 493

Peacock, J. A., \& Wall, J. V. 1982, MNRAS, 198, 843

Readhead, A. C. S., Taylor, G. B., Pearson, T. J., \& Wilkinson, P. N. 1996, ApJ, 460, 634

Spencer, R. E., Schilizzi, R. T., Fanti, C., et al. 1991, MNRAS, 250, 225

Taylor, G. B. 1998, ApJ, 506, 637 